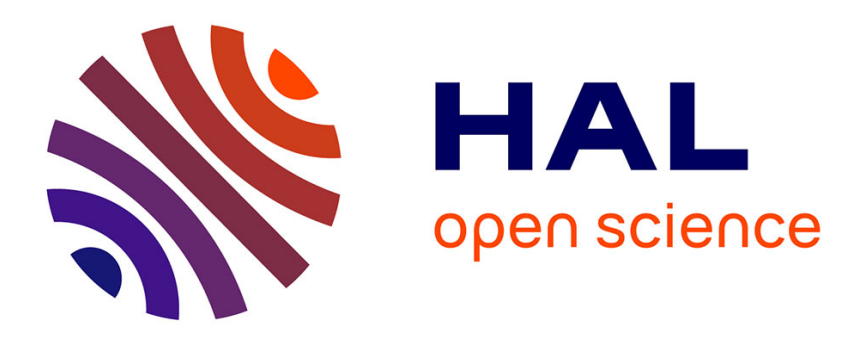

\title{
A Two-Phase Online Prediction Approach for Accurate and Timely Adaptation Decision
}

\author{
Chen Wang, Jean-Louis Pazat
}

\section{To cite this version:}

Chen Wang, Jean-Louis Pazat. A Two-Phase Online Prediction Approach for Accurate and Timely Adaptation Decision. International Conference on Service Computing, IEEE, Jun 2012, honolulu, Hawaii, United States. hal-00705289

\section{HAL Id: hal-00705289 \\ https://hal.inria.fr/hal-00705289}

Submitted on 7 Jun 2012

HAL is a multi-disciplinary open access archive for the deposit and dissemination of scientific research documents, whether they are published or not. The documents may come from teaching and research institutions in France or abroad, or from public or private research centers.
L'archive ouverte pluridisciplinaire HAL, est destinée au dépôt et à la diffusion de documents scientifiques de niveau recherche, publiés ou non, émanant des établissements d'enseignement et de recherche français ou étrangers, des laboratoires publics ou privés. 


\title{
A Two-Phase Online Prediction Approach for Accurate and Timely Adaptation Decision
}

\author{
Chen Wang and Jean-Louis Pazat \\ IRISA/INRIA, Campus de Beaulieu, 35042, Rennes Cedex, France \\ Email: \{chen.wang, jean-louis.pazat\}@inria.fr
}

\begin{abstract}
A Service-Based Application (SBA) is built by defining a workflow that composes and coordinates different Web services available via the Internet. In the context of on-demand SBA execution, suitable services are selected and integrated at runtime to meet different non-functional requirements (such as price and execution time). In such dynamic and distributed environment, an important issue is to guarantee the end-toend Quality of Service (QoS). As a consequence, SBA provider is required to monitor each running SBA instance, analyze its runtime execution states, then identify proper adaptation plans if necessary, and finally apply the relative countermeasures. One of the main challenges is to accurately trigger the adaptation process as early as possible.

In this paper, we present a two-phase decision approach that can accurately analyze the adaptation needs for on-demand SBA execution model. Our approach is based on the online prediction techniques: an adaptation decision is determined by predicting an upcoming end-to-end QoS degradation through two-phase evaluations. Firstly, the end-to-end QoS is estimated at runtime based on monitoring techniques; if a $\mathrm{QoS}$ degradation is tent to happen, in the second phase, both static and adaptive strategies are introduced to assess whether it is the best timing to draw the final adaptation decision. Our approach is evaluated and validated by a series of realistic simulations.
\end{abstract}

Keywords-SBA; adaptation decision; SLA; quality prediction; classification;

\section{INTRODUCTION}

Service-Oriented Architecture (SOA) is adopted today by many enterprises as a flexible solution for building ServiceBased Applications (SBA). An SBA is a dynamic and distributed software system that integrates and coordinates a set of third-party Web services (named as the constituent services) accessible over the Internet. In this scenario, the SBA's endto-end Quality of Service (QoS) is determined by the QoS of all constituent services. For example, the execution time of an SBA instance depends on how fast each constituent service responds. However, in this loosely coupled environment, the execution of SBA may fail, or fail to meet the required quality level: a constituent service may take longer time to respond due to the network congestion; moreover, infrastructure failures can cause a service completely responseless.

In this context, Service Level Agreement (SLA) plays an important role as a guarantee of non-functional quality. SLA is a mutually-agreed contract between service requester and provider, which dictates the expectations as well as obligations in regards to how a service is expected to be provided: on one hand, the expected quality level is formulated by specifying agreed target values for a collection of QoS attributes; on the other hand, some penalty measures are defined in case of failing to meet these quality expectations. SLA violations can lead to some undesirable results, such as reputation degradation and penalty payment. To prevent SLA violation, it is essential for SBA providers to guarantee the end-to-end QoS by taking adaptation countermeasures if needed.

One of the key challenges is to determine the need for adaptation in order to draw accurate adaptation decisions. All existing approaches can be classified into two categories: offline analysis [1], [2], [3] and online prediction [4], [5], [6]. Offline approaches can decide when and how to improve the end-to-end quality of SBA by reasoning the causes of the past SLA violations. The adaptation aims at preventing SLA violations for the future executions rather than the ongoing ones. By contrast, online approaches predict an upcoming SLA violation, and thereby decide to trigger preventive adaptation in order to improve the QoS of the running execution instance before the SLA violation really happens.

This paper investigates an online prediction approach for on-demand executions of SBA. In this context, in response to different non-functional preferences and constraints (e.g. price and execution time), each constituent service is selected at runtime from a set of functional-equivalent candidates with different QoS [7]. As a result, any two distinct executions are instantiated with different configurations, defined as both local and global QoS expectations. Therefore, it is challenging to accurately predict potential SLA violations for all running SBA instances with irrelevant configurations.

In this paper, we introduce a two-phase online prediction approach to decide the best adaptation timing for on-demand executions of SBA. The first phase suspects an SLA violation by predicting an upcoming end-to-end QoS degradation; the prediction is based on the monitoring techniques and the estimation on the future execution. Later in the second phase, both static and adaptive strategies are introduced to predict the reliability of the suspicion in order to decide whether it is necessary to trigger preventive adaptations. Our approach is evaluated by a series of realistic simulations, the results show that our approach can determine both accurate and timely runtime adaptation decisions for on-demand SBA executions.

The rest of paper is organized as follows: Section II provides the background of our problem. In Section III, some of the main existing decision approaches are discussed and our approach is introduced. Later, Section IV and Section V 


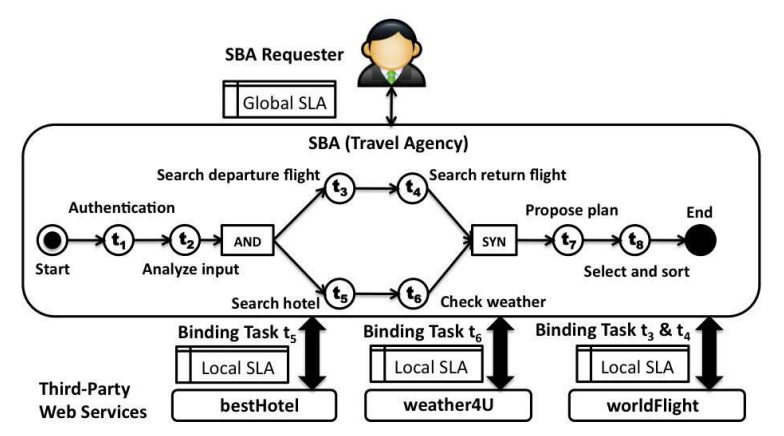

Fig. 1. Illustrative Example: Travel Agency

present respectively the two phases of our approach in depth. In Section VI, the performance of our approach is studied based on a set of realistic simulations. Finally, the conclusion and the future work are addressed in Section VII.

\section{BACKGROUND}

\section{A. Illustrative Example}

In this paper, we use a travel agency as an illustrative example to present our approach. As shown in Figure 1, an SBA is implemented to propose traveling plans for its clients. A request composes a set of parameters such as identification information (e.g. email address and password), dates of traveling and the destination. In order to respond to client's requests, a workflow is defined to coordinate a collection of interrelated tasks: firstly, the client's identity is verified by task $t_{1}$ and the inputs from the requester are validated and analyzed by task $t_{2}$. Then, the execution is diverged into two parallel execution branches: task $t_{3}$ and task $t_{4}$ look for the round-trip flight tickets from all airline companies. Concurrently, task $t_{5}$ searches available hotels in the destination city, and the weather information is provided by task $t_{6}$. Both branches converge before task $t_{7}$, which generates all propositions of traveling plans based on the client's criterion, such as total budget. Finally, task $t_{8}$ sorts all the propositions according to the client's preferences and returns the results.

The SBA requires service collaboration across enterprise boundaries: each task can be bound to either an internal service (e.g. task $t_{1}, t_{2}, t_{7}, t_{8}$ ) or an external one provided by the thirdparty enterprises (e.g. task $t_{3}, t_{4}, t_{5}, t_{6}$ ). Constituent services are on demand selected at runtime: as an example, for premium clients, fast services are selected and bound in order to return the propositions of traveling plans as quickly as possible; on the other side, for normal clients, cheaper and slower services are used and the execution may take respectively longer time.

\section{B. Global and Local SLA}

As introduced, the SLA defines both aspects of expected QoS and penalty measures. First of all, the definition and execution of penalty is beyond the interests of this paper. Furthermore, we assume that a set of QoS attributes can be considered as deterministic: their values cannot be changed at runtime once negotiated (e.g. price). Accordingly, the SLA violation can be only caused by non-deterministic QoS attributes, whose real values are affected by the distributed and
TABLE I

GLOBAL \& LOCAL SLAS

\begin{tabular}{|l|l|l|l|l|l|l|l|l|l|}
\hline & $t_{1}$ & $t_{2}$ & $t_{3}$ & $t_{4}$ & $t_{5}$ & $t_{6}$ & $t_{7}$ & $t_{8}$ & SBA \\
\hline time (ms) & 600 & 800 & 1500 & 1500 & 1800 & 1000 & 1200 & 1000 & 6800 \\
\hline
\end{tabular}

Fig. 2. MAPE Control-Feedback Loop

dynamic runtime environment, such as response time. For the reason of simplicity, an SLA is therefore modeled as a set of target values for only non-deterministic QoS attributes. As a proof on concept, our discussion will only focus on the response time in the remainder of this paper.

The SBA plays the roles of service provider as well as consumer. For both roles, it negotiates an SLA with each of its counterparts, as shown in Figure 1: the SLA between the SBA and each constituent service is defined as local $S L A$, and the one negotiated with the SBA requester is defined as global SLA. A local SLA reflects the expected time consumption for executing the corresponding task $t_{i}$, denoted as $l s l a\left(t_{i}\right)=<q_{t}\left(t_{i}\right)>$, whereas the global SLA dictates the expected end-to-end execution time of SBA, denoted as $g s l a=\left\langle g c_{t}\right\rangle$. Obviously, the definition of the global SLA depends on the local ones. As an example, Table I lists the expected execution time defined in both global and local SLAs for an instance of the illustrative example shown in Figure 1.

\section{Prevention of Global SLA Violation}

In order to enhance the reputation and to avoid penalties, it is mandatory for service providers to prevent global SLA violation for every running execution instance. The general solution for runtime prevention of SLA violation is to implement the MAPE control-feedback loop (Monitor-AnalyzePlan-Execute) [8], as depicted in Figure 2: 1) Monitor: firstly, each execution instance of SBA is monitored by intercepting communication messages in order to collect a series of events; 2) Analyze: these events are used to evaluate the quality state of a running execution instance and to analyze the need for adaptation; 3) Plan: once an adaptation decision is determined, a suitable adaptation plan (e.g. a list of actions to improve the end-to-end quality) is identified; 4) Execute: finally the relative countermeasures are applied to the ongoing SBA instance.

\section{Problem Definition}

One of the key challenges to efficiently implement the MAPE loop is to accurately draw adaptation decision (Analyze step). Our research work studies an online prediction approach to analyze the need for adaptation by forecasting whether the SLA is tent to be violated in the future. An eligible online prediction approach has to meet the following requirements (challenges): 1) Effectiveness. An effective approach can successfully predict as many SLA violations as possible. 2) Precision. An effective approach might not be precise due to many false predictions, which will lead to unnecessary adaptations and thereby bring additional cost and complexity: on one hand, runtime adaptation is costly since more resources 
are required to identify and to execute an adaptation plan; on the other hand, the time consumption of a runtime adaptation process may potentially delay the execution of SBA. 3) Timing. It is desirable to decide as early as possible: late decisions are usually precise but less useful, since the best adaptation opportunities might be missed, and the benefit of preventive adaptation is diminished. 4) Efficiency. The decision algorithm must be efficient (fast decision) in order to meet the critical time constraint at runtime.

\section{Related Work And Motivation}

\section{A. Related Work}

The existing online prediction approaches in the literature can forecast SLA violations caused by either functional failures or non-functional deviations. In the former case, the recovery from functional failures requires extra execution time and additional cost, which can lead to global SLA violations. Some research work use online testing techniques to test all constituent services in parallel to the execution of an SBA instance. By this means, an upcoming functional failure can be forecasted before its real occurrence. [9] presents the PROSA framework, which defines key activities to initiate online testing either on the binding level or on the service composition level, and thereby proactively triggers the adaptation process. [10] investigates how to guarantee functional correctness of conversational services. The authors propose a novel approach to enables proactive adaptations through just-in-time testing. Online testing approach is helpful to detect potential functional failures but it can hardly be aware of the deviation of the endto-end QoS. Furthermore, it requires each consistent service to provide a test mode (e.g. free interfaces for testing).

Our research work belongs to the latter case, which predicts SLA violation by asserting non-functional deviations that might happen at the end of the execution (e.g. delay). Some research work use runtime verification techniques to determine the necessity of adaptation and to trigger preventive adaptation. In [6], the authors introduce SPADE approach: after the execution of each task, if the local SLA is violated, SPADE uses both monitored data and the assumptions to verify whether the global SLA can be still satisfied. If it reveals that the global SLA is tent to be violated, the adaptation is accordingly triggered. However, early verifications are largely based on the assumptions rather than monitored data, thus they are inaccurate and can lead to many unnecessary adaptations.

Other research work use machine learning techniques in order to provide precise predictions and avoid unnecessary adaptations. In [4], a set of concrete points are defined in the workflow as checkpoints. Each checkpoint is associated with a predictor, which is implemented by a regression classifier. When the execution of the workflow reaches to a checkpoint, the corresponding predictor is activated and uses the knowledge learned from past executions to predict whether the global SLA will be violated. This work is extended in [5] by proposing the PREvent framework, which integrates eventbased monitoring, runtime prediction of SLA violations and

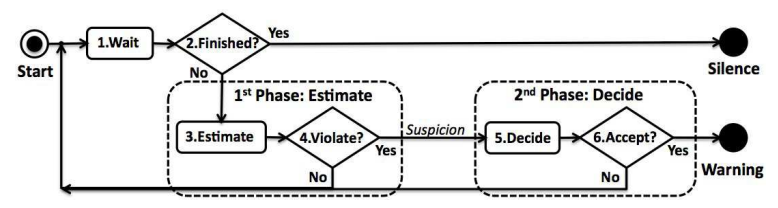

Fig. 3. 2-Phase Online Prediction Approach

automated runtime adaptations. Such checkpoint-based prediction approach has some limitations: firstly, some misbehavior (e.g. huge delay) between two checkpoints cannot be handled in time. In the meantime, the best adaptation opportunity might be lost. Furthermore, poorly selected checkpoint(s) may lead to undesirable results, such as unnecessary adaptations. But the selection of optimal checkpoints is complicated and challenging, especially for complex workflows.

\section{B. Our Approach: Two-Phase Decision Approach}

In order to provide both accurate and timely predictions of SLA violation, we propose a two-phase online prediction approach. As shown in Figure 3, an adaptation decision is determined through the following steps: 1) listen to the events emitted from the Monitor component (refer to Figure 2), once the execution of a task $t_{i}$ is completed, go to step 2. 2) If the execution of workflow has not yet been finished, go to step 3; otherwise, the algorithm is terminated with the state silence, which means that during the entire execution, no SLA violation has been predicted (no adaptation decision has been made). 3) Estimate the values of QoS attributes defined in the global SLA (e.g. execution time) based on the monitored data and the estimation of the execution in the future. 4) Compare the estimated value with the target value defined in the global SLA, if a violation is tent to happen, a suspicion of SLA violation is reported and go to step 5; otherwise, return to step 1. 5) Evaluate the trustworthy level of the suspicion in order to decide whether to accept or to neglect this suspicion. 6) If the suspicion is accepted, our approach terminates with the state warning by predicting an upcoming SLA violation and drawing the adaptation decision; otherwise, the suspicion is neglected and go back to step 1 .

The core of our approach is two-phase evaluations: the estimation phase (step 3,4) evaluates whether the global SLA is tent to be violated and the decision phase (step 5, 6) evaluates how likely the suspected violation will really happen. An additional evaluation can bring more precise adaptation decisions since all inaccurate early suspicions can be neglected in the decision phase. Additionally, without the limitation of the predefined checkpoints, it is possible to react to any misbehavior in time. In the following, Section IV and Section V will use the execution time as an example to highlight respectively these two phases.

\section{Estimation Phase}

To estimate the end-to-end execution time of a running SBA instance, two kinds of information are required: 1) the measured execution time of the tasks whose executions have already been completed; 2) the probable time consumption of 
uncompleted tasks, including the tasks that are being executed as well as the ones whose executions have not been started yet. Based on the monitoring techniques [5], we assume that the former information is known at the time of estimation, which is accessible from an internal database. In this section, we firstly highlight how to estimate local execution time for uncompleted tasks, and then we introduce an efficient tool for rapid estimation of the global execution time.

\section{A. Estimation of Local Execution Time}

The local execution time of a task $t_{i}$ depends on how fast the corresponding constituent service $S_{B}\left(t_{i}\right)$ responds. As a result, some research work [5] propose to use arithmetic mean value of the last $n$ measured response time of $S_{B}\left(t_{i}\right)$ as the estimation of the local execution time for $t_{i}$, denoted as $q_{E}\left(t_{i}\right)$. However, the performance of this method is affected by the outliers. Suppose that the last 10 measures of response time are: $940 \mathrm{~ms}, 1,020 \mathrm{~ms}, 1,050 \mathrm{~ms}, 1,000 \mathrm{~ms}, 970 \mathrm{~ms}, 1,100 \mathrm{~ms}$, $1,020 \mathrm{~ms}, 24,060 \mathrm{~ms}, 960 \mathrm{~ms}, 980 \mathrm{~ms}$. The arithmetic mean is $3,310 \mathrm{~ms}$, which cannot properly reflect the probable response time of $S_{B}\left(t_{i}\right)$. In addition, this method cannot be used when there is no (sufficient) historical information. For example, in the context of on-demand SBA execution, a task may bind to a service that has never been invoked before.

We provide both dynamic and static methods for local estimation. To estimate the response time of $S_{B}\left(t_{i}\right)$, SBA provider is required to record the response time of all constituent services that have been invoked before. Dynamic methods look into the past records for the information about $S_{B}\left(t_{i}\right)$. If enough historical information is found, the approaches presented in [11] can be used to firstly detect and remove the outliers before computing the arithmetic mean. A more efficient alternative solution is to directly use the median value of the last $\mathrm{n}$ measures. In the previous example, the median value is $1000 \mathrm{~ms}$ : five measures are less or equal to it and five are greater, including the outlier. In case of no (sufficient) historical information about $S_{B}\left(t_{i}\right)$, the static method is automatically activated, which uses the target value defined in the local SLA as the estimation $\left(q_{E}\left(t_{i}\right)=q_{t}\left(t_{i}\right)\right)$. In this case, it is straightforward to trust the service provider.

\section{B. Estimation of Global Execution Time}

Having the runtime knowledge on the local execution time of each task $t_{i}$, defined as $q_{L}\left(t_{i}\right)$, the global execution time of a running SBA instance can be estimated by using aggregation functions [12] $\left(q_{L}\left(t_{i}\right)\right.$ equals to the measured execution time for completed tasks and equals to $q_{E}\left(t_{i}\right)$ for uncompleted ones). However, runtime aggregation is costly and time-consuming, especially for complex and unstructured workflows. We introduce the Program Evaluation and Review Technique (PERT) [13] as an efficient tool for rapid runtime estimation of global execution time. PERT was originally developed for planning, monitoring and managing the progress of complex projects. In our approach, PERT is used to manage the workflow execution and to facilitate decision making. It maintains some additional information for each task $t_{i}$ :

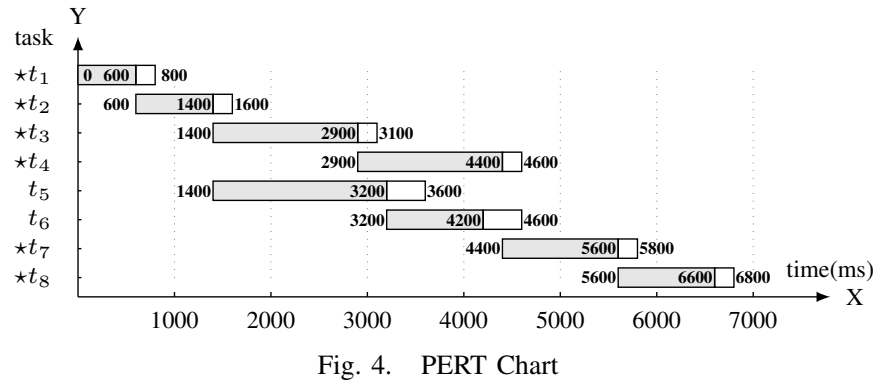

- The Expected Start Time (EST): $T_{E}\left(t_{i}\right)$. This is the expected time by which the execution of task $t_{i}$ can start. $T_{E}\left(t_{i}\right)=\max \left\{T_{E}\left(t_{j}\right)+q_{L}\left(t_{j}\right) \mid t_{j}\right.$ is the direct precedent of $\left.t_{i}\right\}$, For the first task (start) $t_{s}, T_{E}\left(t_{s}\right)=0$.

- The Latest Finish Time (LFT): $T_{L}\left(t_{i}\right)$. This is the latest time by which the task $t_{i}$ can finish without causing the delay of the ongoing execution instance. $T_{L}\left(t_{i}\right)=$ $\min \left\{T_{L}\left(t_{k}\right)-q_{L}\left(t_{k}\right) \mid t_{k}\right.$ is the direct successor of $\left.t_{i}\right\}$. For the last task (end) $t_{n}, T_{E}\left(t_{n}\right)=g c_{t}$, which is the target value defined in the global SLA.

- The slack time: $S\left(t_{i}\right)$. This is the maximum delay for executing the task $t_{i} . S\left(t_{i}\right)=T_{L}\left(t_{i}\right)-T_{E}\left(t_{i}\right)-q_{L}\left(t_{i}\right)$.

- The critical path CP. A path is a sequential execution of tasks from the beginning to the end of a workflow. $\mathrm{CP}$ is a path with all tasks having the least slack time.

The PERT chart is initially constructed based on both global and local SLAs $\left(q_{L}\left(t_{i}\right)=q_{t}\left(t_{i}\right)\right)$, all the information is then presented on an X-Y chart. As an example, for the execution instance of the illustrative example with all local and global SLAs given in Table I, the corresponding PERT chart is computed and depicted in Figure 4. The X-axis represents the accumulated execution time, while the Y-axis is the list of tasks that are composed in the workflow. Each task $t_{i}$ is represented by a single horizontal bar which starts from $T_{E}\left(t_{i}\right)$ and ends with $T_{L}\left(t_{i}\right)$. The length of the bar corresponds to the maximum acceptable duration for executing a task, under the assumption that all the other tasks complete on schedule. A bar is composed of two parts, the solid part represents $q_{L}\left(t_{i}\right)$ and the hollow part reflects $S\left(t_{i}\right)$. Finally, the tasks on the critical path is marked by a star $(\star)$.

In order to reflect the most up-to-date runtime execution state, the PERT chart can be updated when new local knowledge $q_{L}\left(t_{i}\right)$ is available (e.g. newly measured execution time of a just-completed task). Please note that the PERT chart only needs to be partially reconstructed by recomputing some of the above-mentioned information (e.g. the completed tasks do not need to be recomputed), which can be seen as the adjustments of some bars along the $\mathrm{X}$-axis. The update can be triggered either periodically, or based on checkpoints or events (e.g. after each "invoke" event). Anyhow, the reconstruction of chart can be performed in parallel with the service invocations, thus it will not bring extra time consumption.

By using the PERT chart, the estimation of global execution time is simplified. When a task $t_{i}$ is finished, the real accumulated execution time at this moment can be measured, denoted as $A T\left(t_{i}\right)$. If $A T\left(t_{i}\right)>T_{L}\left(t_{i}\right)$, the global execution 
time is estimated to be violated by $A T\left(t_{i}\right)-T_{L}\left(t_{i}\right)$, and a global SLA violation is accordingly suspected. A suspicion of violation is represented by a pair, denoted as $S=\left\langle T_{D}, t_{i}>\right.$, which means that after executing task $t_{i}$, a delay of $T_{D}$ is estimated $\left(T_{D}=A T\left(t_{i}\right)-T_{L}\left(t_{i}\right)\right)$. In this way, instead of running complex aggregation function, the estimation of global SLA requires only one comparison operation.

\section{Decision Phase}

In this section, both static and adaptive decision strategies are introduced to evaluate the trustworthy level of a suspicion in order to identify the need for the preventive adaptation.

\section{A. Weight of a Suspicion}

In the context of on-demand SBA execution, two suspicions with the same attributes $\left(T_{D}\right.$ and $\left.t_{i}\right)$ reported by two distinct SBA instances may have different significances. With different configurations, "after task $t_{i}$ " refers to the different degrees on the completion of workflow execution. Using our illustrative example, $S_{1}$ and $S_{2}$ are both reported after task $t_{7}$ with the same estimated delay of $350 \mathrm{~ms}$; but for $S_{1}, 90 \%$ of the global expected execution time $\left(g c_{t}\right)$ is consumed after executing $t_{7}$, whereas only $60 \%$ for $S_{2}$. Obviously, $S_{1}$ is stronger than $S_{2}$ since a delay is more likely to happen in the end.

In order to model the significance of a suspicion $S$ for on-demand executions of SBA, we introduce the concept of weight, denoted as $\tau_{s} . \tau_{s}$ is defined by a decimal between 0 and 1: greater value indicates a stronger suspicion. An earlier suspicion is often less accurate, because it is based more on the estimated values than on the measured values; accordingly it has a lower weight. Along with the execution, since more and more local estimations are updated with measured values, a later suspicion becomes more accurate and has a respectively higher weight. In our approach, the weight of a suspicion $S$ is expressed by the percentage of execution that is supposed to be completed at the moment, defined as $\tau_{s}(S)=T_{L}\left(t_{i}\right) / g c_{t}$, remember that $T_{L}\left(t_{i}\right)$ denotes the LFT of task $t_{i}$ and $g c_{t}$ is the expected global execution time defined in the global SLA.

We provide three static strategies and an adaptive strategy to evaluate a suspicion $S$ using the estimated delay $T_{D}$ and its weight $\tau_{s}(S)$. Static strategies compute the maximum allowed delay $\max _{d}$ with respect to $\tau_{s}(S)$ using predefined evaluation functions. If $T_{D}$ is greater than $\max _{d}$, the suspicion is considered as strong enough and an adaptation decision is determined; otherwise, it is neglected. By contrast, adaptive strategy uses machine learning techniques to build a classifier that learns the knowledge from the past suspicions in order to predict whether or not the current suspicion will really happen.

\section{B. Static Decision Strategies}

1) Qualitative strategy: The idea is to neglect all the early suspicions since they are considered as inaccurate. By specifying a weight threshold $\rho_{w}$, only the suspicions with $\tau_{s}>\rho_{w}$ will be accepted to report a warning of violation. Thus, its evaluation function is a step function, as depicted by $f_{1}$ in Figure 5: $\max _{d}$ equals to $+\infty$ if $\tau_{s}$ is smaller than $\rho_{w}$

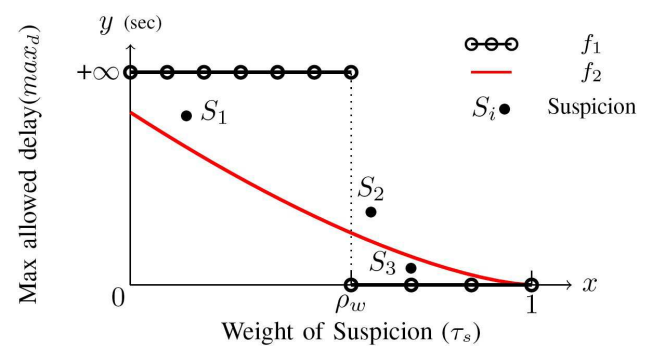

Fig. 5. Evaluation Functions of Static Decision Strategies

and equals to 0 otherwise. Figure 5 also gives three sample suspicions with different weights and estimated delays: using qualitative strategy, $S_{1}$ will be neglected whereas both $S_{2}$ and $S_{3}$ will be accepted.

2) Quantitative strategy: The main limitation of qualitative strategy is that the adaptation cannot be triggered until a certain percentage of workflow has been executed, despite the fact that a huge delay may arise at the beginning of the execution (e.g. $S_{1}$ ). In order to react to such problem as early as possible, quantitative strategy evaluates a suspicion with the consideration of both $\tau_{s}(S)$ and $T_{D}$. In this case, the SBA provider specifies an evaluation function based on his/her own experience, such as $f_{2}$ defined in Figure 5. Using $f_{2}, S_{1}$ and $S_{2}$ will lead to adaptation decisions while $S_{3}$ will not.

3) Hybrid strategy: The quantitative strategy may not detect slight delays when the execution is approaching to the end, such as $S_{3}$. In this case, a slight delay might finally lead to a great penalty due to SLA violation. The hybrid strategy is more critical: by specifying $\rho_{w}$, if $\tau_{s}(S)$ is greater than $\rho_{w}$, the qualitative strategy is applied ( $S$ will be absolutely accepted); otherwise, the quantitative strategy is applied. Thus, its evaluation function follows $f_{2}$ when $\tau_{s}<\rho_{w}$ and follows $f_{1}\left(\max _{d}=0\right)$ otherwise. By using hybrid strategy, all three suspicions in Figure 5 will be accepted.

\section{Adaptive Decision Strategy}

Static decision strategies are useful when insufficient historical information is available. However, from the long-run perspective, it has the following limitations: first of all, it is a challenging task for the SBA provider to manually identify a suitable evaluation function based on his/her past experiences: sometimes such experience is hard to be expressed using a regular function. Additionally, once defined, the evaluation function cannot be self-adjusted (can be manually modified by SBA provider) in order to improve the quality of decision.

The adaptive strategy models adaptation decision as a classification problem. The correctness of a suspicion $\left(C_{S}\right)$ can be evaluated when the relative execution terminates (suppose no adaptation action is really executed): if the global SLA is really violated, the suspicion is proved as correct $\left(C_{S}=\right.$ true); otherwise, it is marked as a false one $\left(C_{S}=\right.$ false $)$. As a result, at the end of each execution, a set of suspicion records can be created based on all reported suspicions and their correctness. A suspicion record is described by two numeric attributes (estimated delay and its corresponding weight) and a categorical attribute defined as class (can be true or false), 


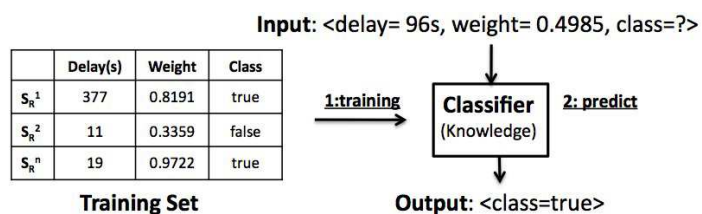

Fig. 6. Adaptive Decision Strategy

denoted as $S_{R}=<T_{D}, \tau_{s}(S), C_{S}>$. All historical suspicion records are organized into a training dataset, as illustrated in Figure 6. The dataset is often depicted as a table, with each row representing a suspicion record. Based on machine learning technique [14], a classifier is built to progressively learn the knowledge from past experiences. The knowledge is a specific algorithm that determines the class of a new suspicion based on its attributes. Once a suspicion is reported and it is classified as correct $\left(C_{S}=\right.$ true $)$, the adaptation decision is accordingly made; otherwise, this suspicion is neglected.

Since the space is limited, we will not provide the details about data cleaning as well as how to learn and represent the knowledge. Interested readers can refer to [14], [15]. The classifier is required to be retrained to improve the prediction quality. The retraining can be carried out in one of the following ways: 1 ) after every $N$ predictions, 2 ) periodically (after a fixed duration), 3) on-demand by the SBA provider.

\section{EXPERIMENTAL RESULTS}

\section{A. Experiment Setup}

Our approach is evaluated and validated by a set of experiments built on a realistic simulation model, since real implementation is costly, which requires to implement the entire MAPE control loop as well as dealing with some other challenging problems of on-demand SBA execution, such as service selection, or interface mismatches.

1) Realistic simulation model: For each constituent service, we are only interested in how it responds rather than what it responds. Therefore, instead of invoking real-world Web services, each task is bound to a virtual service, which only simulates the non-functional aspects of a service invocation (e.g. response time). In our experiments, 100 virtual services are created based on the realistic QoS datasets provided by [16], which record the non-functional performances (such as response time, throughput, etc.) of a large number of real-world service invocations. A virtual service collects all the invocation records from the same requester to the same Web service. By using these records, each virtual service defines two methods: 1) simulate() randomly selects one of the past records to simulate the non-functional aspects of an invocation; 2) getExpectedRT() determines the expected response time by specifying a percentage threshold $\phi$, which indicates the percentage of past invocations which can respond within the expected value. In our experiments, in order to create a scenario with high violation rate, $\phi$ is set to $0.6(40 \%$ possibility of local SLA violations for each virtual service).

2) Simulate an execution of SBA: An execution of SBA is simulated through three stages: in the first stage, an SBA instance is created by binding each task $t_{i}$ to a randomly
TABLE II

CONTINGEnCy TABLE

\begin{tabular}{|l|l|l|l|}
\hline & Real: violated & Real: not violated & Sum \\
\hline $\begin{array}{l}\text { Prediction: violated } \\
\text { (warning) }\end{array}$ & $\begin{array}{l}\text { True Positive (TP) } \\
\text { (correct warning) }\end{array}$ & $\begin{array}{l}\text { False Positive (FP) } \\
\text { (false warning) }\end{array}$ & $\begin{array}{l}\text { Positive } \\
(\mathrm{P})\end{array}$ \\
\hline $\begin{array}{l}\text { Prediction: not violated } \\
\text { (silence) }\end{array}$ & $\begin{array}{l}\text { False Negative (FN) } \\
\text { (false silence) }\end{array}$ & $\begin{array}{l}\text { True Negative (TN) } \\
\text { (correct silence) }\end{array}$ & $\begin{array}{l}\text { Negative } \\
\text { (N) }\end{array}$ \\
\hline Sum & Violations (V) & Compliance (C) & Total (T) \\
\hline
\end{tabular}

selected virtual service, denoted as $v s\left(t_{i}\right)$, and the relative local SLA is generated by completing a template with the expected response time of $v s\left(t_{i}\right)$. Then, the global SLA is generated by computing the expected end-to-end execution time using aggregation functions [12]. Finally, based on both local and global SLAs, a PERT chart is constructed.

The second stage simulates the execution of this SBA instance. First of all, each selected virtual service $v s\left(t_{i}\right)$ simulates the response time as the real execution time of task $t_{i}$. Then, by running aggregation functions along all execution paths, the real accumulated execution time by which the execution of task $t_{i}$ is completed can be computed, denoted as $A T\left(t_{i}\right)$. Next, a collection of "receive" events are created with the corresponding timestamp, denoted as Recv $=\left\langle t_{i}, A T\left(t_{i}\right)>\right.$.

Finally in the third stage, these "receive" events are sorted by the timestamp and then sequentially processed: firstly, if $A T\left(t_{i}\right)>T_{L}\left(t_{i}\right)$, a set of predictors are activated to make adaptation decision based on different strategies. A predictor is a Java object that implements a specific decision strategy. After the prediction, the PERT chart is updated and the static method is used for estimations of local execution time.

\section{B. Evaluation Metrics}

Contingency table metrics [17] are used to investigate how accurately a decision approach works. An adaptation decision approach can terminate with two possible states: warning or silence (refer to Figure 3). Using the contingency table, as shown in Table II, a warning is defined as a positive decision $(\mathrm{P})$, which asserts that the global SLA will be violated in the near future; by contrast, a silence is formally named as a negative decision $(\mathrm{N})$ which decides that no adaptation was needed for the entire duration of the execution. In order to evaluate the quality of decision, no adaptation plan is really identified and executed. For a positive decision, if a violation is really occurred in the end, it is proved to be a true positive (TP); otherwise, it is a false positive (FP). Similarly, a negative decision can be either a true negative (TN) if no violation really happens at the end of execution, or else a false negative (FN). Based on the contingency table, different evaluation metrics are defined as follows:

- Accuracy $(a)$. It is the ratio of all correct decisions to the number of all decisions: $a=\frac{T P+T N}{T P+T N+F P+F N}=\frac{T P+T N}{T}$.

- Precision $(p)$. It is the ratio of all correct warnings to the number of all warnings, $p=\frac{T P}{T P+F P}$.

- Effectiveness $(e)$. It is the ratio of all correct silences to the number of all silences: $e=\frac{T N}{T N+F N}$.

- Decision Time $(d t)$. Only positive decisions have $d t$. It is measured by the maximum number of tasks that have already been completed on different execution paths. 
TABLE III

EXPERIMENTAL RESULTS: EVALUATION OF DECISION TIMING

\begin{tabular}{|l|l|l|l|l|l|l|l|l|l|l|}
\hline Metrics & $P_{1}$ & $P_{2}$ & $P_{3}$ & $P_{4}$ & $P_{5}$ & $P_{6}$ & $P_{7}$ & $P_{8}$ & $P_{9}$ & $P_{10}$ \\
\hline TP & 191 & 243 & 171 & 211 & 165 & 191 & 397 & 441 & 406 & 359 \\
\hline FP & 156 & 146 & 59 & 45 & 61 & 41 & 39 & 0.0 & 162 & 79 \\
\hline FN & 250 & 198 & 270 & 230 & 276 & 250 & 44 & 0.0 & 35 & 82 \\
\hline TN & 403 & 413 & 500 & 514 & 498 & 518 & 559 & 520 & 397 & 480 \\
\hline a & 0.59 & 0.66 & 0.67 & 0.73 & 0.66 & 0.71 & 0.92 & 1.0 & 0.8 & 0.84 \\
\hline e & 0.43 & 0.55 & 0.39 & 0.48 & 0.37 & 0.43 & 0.90 & 1.0 & 0.92 & 0.81 \\
\hline p & 0.72 & 0.74 & 0.90 & 0.92 & 0.89 & 0.93 & 0.93 & 1.0 & 0.71 & 0.86 \\
\hline dt & 1 & 2 & 3 & 4 & 3 & 4 & 5 & 6 & 2.95 & 4.0 \\
\hline
\end{tabular}

\section{Experiment 1: Evaluation of the Best Decision Time}

In this experiment, the best decision time is evaluated by disabling the decision phase, thereby the adaptation decision is determined once an SLA violation is suspected. The evaluation is based on the illustrative example defined in Figure 1: after each task $t_{i}(1 \leq i \leq 8)$, a checkpoint $C_{i}$ is defined as a possible decision time point. 8 predictors are created based on a single checkpoint: predictor $P_{i}(1 \leq i \leq 8)$ can decide only when the execution of workflow reaches to checkpoint $C_{i}$. Please note that the decisions of $P_{8}$ must be definitely accurate because it decides after the completion of workflow execution. In addition, two predictors are defined based on multiple checkpoints: $P_{9}$ is activated at both $C_{2}$ and $C_{7}$ while $P_{10}$ can decide at either $C_{4}$ or $C_{6}$. We run 1000 simulations, Table III summarizes the performance of all predictors.

1) Decisions based on a single checkpoint: The results reveal that, by using a single checkpoint, it is hard to draw both early and accurate adaptation decision. First of all, as we discussed in this paper, early decisions are less accurate: due to the large number of FP and FN decisions, $P_{1}$ and $P_{2}$ result in lower accuracy, precision as well as effectiveness. By contrast, as the most part of workflow has been executed, $P_{7}$ performs largely better but its decisions come too late to carry out effective preventive adaptations. Furthermore, the other four predictors are based on the checkpoints located on two parallel execution branches, and the critical path can be only determined at runtime due to on-demand service selection. If a task (checkpoint) is not on the critical path, it has thus a larger slack time and a longer delay can be tolerated. Therefore, from its local perspective, the execution is considered as running well and no warning will be reported. That explains why $P_{3}$, $P_{4}, P_{5}$ and $P_{6}$ have less FP decisions but a tremendous number of FN decisions, which lead to a poor performance on average.

2) Decisions based on multiple checkpoints: An additional checkpoint brings another chance to report (either true or false) warnings of SLA violations. Take $P_{9}$ for example, if a violation is failed to be predicted at $C_{2}$, it still has chance to be alerted at $C_{7}$; thus, compared to $P_{2}$, the TP number is greatly improved; meanwhile, only several FP decisions are additionally produced. On the other side, compared to $P_{7}$, although $P_{9}$ does get some quality degradation, but the decision time is improved by 2 execution steps. This is the same for $P_{10}$, compared to $P_{4}$ and $P_{6}$, both accuracy and effectiveness are significantly improved. However, as an extreme case, we assume that a predictor $P_{x}$ can draw adaptation decisions at all possible checkpoints. It is similar to runtime verification
TABLE IV

ExPERIMENTAl Results: Evaluate DifFERENT Decision Strategies

\begin{tabular}{|l|l|l|l|l|l|l|l|}
\hline Metrics & $P_{7}$ & $P_{9}$ & $P_{10}$ & $P_{q l}$ & $P_{q t}$ & $P_{h b}$ & $P_{a d}$ \\
\hline $\mathrm{a}$ & 0.91 & 0.82 & 0.84 & 0.94 & 0.95 & 0.92 & 0.94 \\
\hline e & 0.90 & 0.93 & 0.81 & 1.0 & 1.0 & 1.0 & 0.95 \\
\hline p & 0.91 & 0.74 & 0.87 & 0.89 & 0.91 & 0.86 & 0.93 \\
\hline dt & 5 & 3.04 & 4 & 4.2 & 3.9 & 3.8 & 3.8 \\
\hline
\end{tabular}

techniques introduced in Section III: whenever a deviation is estimated, the adaptation decision is made. In this case, any a predictor from $P_{1}$ to $P_{8}$ decides, $P_{x}$ will also decide. Thus, $P_{x}$ cannot have less FP numbers than $P_{1}$. Such a high FP number will result in a poor precision. From the above discussion, we can see that it is challenging to determine the best time for drawing both early and accurate adaptations decisions.

\section{Experiment 2: Evaluation of Our Approaches}

In the second experiment, both static and adaptive decision strategies presented in this paper are evaluated. The qualitative predictor $P_{q l}$ implements qualitative strategy by specifying the weight threshold $\rho_{w}$ to 0.65 . The quantitative predictor $P_{q t}$ defines the evaluation function as $\max _{d}=\left(1-\tau_{s}\right)^{\frac{3}{2}} * g c_{t} * p$, where $\tau_{s}$ is the weight of a suspicion, $g c_{t}$ is the expected execution time of SBA defined in the global SLA, and $p$ is set to $5 \%$. This function can tolerate greater deviation at the beginning of the execution (as $f_{2}$ in Figure 5). Additionally, the hybrid predictor $P_{h b}$ invokes $P_{q t}$ when the weight of suspicion is less than 0.65 , and uses $P_{q l}$ otherwise. Finally, the adaptive predictor $P_{a d}$ implements a set of classifiers based on WEKA machine learning toolkit [15]. All the decisions reported in the first experiment are used to generate a set of suspicion records, which are organized in the dataset file.

During the training phase (Step 1 in Figure 6), all classifiers are used to learn the knowledge from the dataset, and their performances are evaluated by cross-validation. When a new suspicion is reported, the classifier with the best predictive accuracy is then used for the prediction (Step 2 in Figure 6). Each classifier requires at least 300 historical suspicion records (min_training_size $=300$ ); if the dataset contains more than 1000 records, it selects the 1000 most recent items (max_training_size $=1000$ ). The retraining is carried out for every 200 predictions.

Another 1000 executions are simulated to evaluate our approach. As a comparison, the three predictors with the best decision quality in the first experiment, namely $P_{7}, P_{9}$ and $P_{10}$, are also used. The performance of different predictors is shown in Table IV. First of all, we can see that the quality of all static and adaptive decision strategies can be considered on the same level. Please note that: 1) $P_{q l}$ decides later than the other three strategies, since it can decide only when a certain part of workflow has been executed. 2) As introduced, $P_{h b}$ is more critical than $P_{q l}$ and $P_{q t}$. Thus it accepts more suspicions, which can result in a lower precision whereas a higher effectiveness. 3) Static strategies can successfully prevent almost all of SLA violations (luckily, in this experiment, no SLA violation has been missed). Meanwhile, adaptive strategy has a low rate of false silence (5\%). 
TABLE V

ACCURACY

\begin{tabular}{|l|l|l|l|l|}
\hline WF & $P_{q l}$ & $P_{q t}$ & $P_{h d}$ & $P_{a d}$ \\
\hline 1 & 0.93 & 0.93 & 0.91 & 0.96 \\
\hline 2 & 0.90 & 0.94 & 0.89 & 0.95 \\
\hline 3 & 0.91 & 0.93 & 0.89 & 0.97 \\
\hline
\end{tabular}

\begin{tabular}{|l|l|l|l|l|}
\hline WF & $P_{q l}$ & $P_{q t}$ & $P_{h d}$ & $P_{a d}$ \\
\hline 1 & 0.88 & 0.90 & 0.85 & 0.95 \\
\hline 2 & 0.83 & 0.90 & 0.81 & 0.95 \\
\hline 3 & 0.83 & 0.88 & 0.80 & 0.96 \\
\hline
\end{tabular}

Secondly, compared to checkpoint-based predictors (singlestep predictions), the results show that our approach can make more accurate adaptation decisions as early as possible. First of all, the decision quality of our approach can be considered on the same level as $P_{7}$. But our approach improves the decision time by more than one execution step. Secondly, $P_{9}$ decides a little earlier than our approaches (less than one execution step), but our approach has a remarkable improvement in accuracy (over 10\%), effectiveness (5\% higher on average) and precision ( $15 \%$ better). Finally, compare to $P_{10}$, our approaches have almost the same decision time but better accuracy $(\approx 10 \%)$ as well as higher effectiveness $(\approx 15 \%)$.

\section{E. Experiment 3: Evaluations over Different Workflows}

In order to evaluate the performance of our approach over different workflows, we create three fictitious workflows without real meanings: 1) a linear workflow with 9 tasks (a single execution path); 2) a medium workflow with 17 tasks and 6 execution paths; 3) a complex workflow with 30 tasks and 9 execution paths. For each workflow, we firstly simulate 300 executions to initiate the dataset of suspicion records, and then 1000 simulations are carried out by using $P_{q l}, P_{q t}, P_{h b}$ and $P_{a d}$. Table $\mathrm{V}$ and Table VI summarize respectively the accuracy and the precision of different predictors. From the experimental results, we have the following observations: 1) our approach is not limited to a specific workflow and it can perform well for different kinds of workflows; 2) adaptive strategy makes decisions based on the knowledge learned from the past executions, thus its performance can always be maintained at a high level when used for different workflows; 3) the performances of static strategies depend on the predefined evaluation function: as the second experiment demonstrates, a suitable evaluation function can perform as well as adaptive strategy; otherwise, it may get a little performance degradation but it is still fairly good (compared to other approaches).

\section{CONCLUSION AND FUTURE WORK}

This paper discusses an important problem for preventing SLA violation: how to make correct runtime decision to accurately trigger preventive adaptation for on-demand SBA execution. In this paper, we have presented an online prediction approach which draws adaptation decisions through twophase evaluations. Based on a series of realistic simulations, our approach exhibits the following desirable properties: 1) it is able to draw accurate adaptation decisions for different workflows: almost all violations can be successfully predicted and alerted; meanwhile, only few unnecessary adaptations will be triggered; 2) with the same accuracy level, our approach can decide as early as possible; 3) by using static strategies, our approach can still make accurate and timely adaptation decision when no (sufficient) historical information is available.
Our future work will concentrate on providing more flexible runtime management of SBA. First of all, the PERT technique can be used for runtime optimization of SBA instances. For example, by using PERT charts, it is easy to detect large slack time for a task and thus it can rebind to a slower but cheaper service in order to reduce the cost. Furthermore, we are integrating branch prediction techniques into PERT charts, which can help to further reduce unnecessary adaptations. The re-construction of PERT chart will take into account the paths that are most likely to be executed. Finally, having positive results based on the realistic simulations, we are going to implement and integrate our approach into an existing business process management system and evaluate its performance.

\section{ACKNOWLEDGMENT}

The research leading to these results has received funding from the European Communitys Seventh Framework Programme [FP7/2007-2013] under grant agreement 215483 (SCUBE).

\section{REFERENCES}

[1] L. Bodenstaff, A. Wombacher, M. Reichert, and M. Jaeger, "Monitoring dependencies for slas: The mode4sla approach," in IEEE International Conference on Services Computing (SCC '08), july 2008, pp. 21 -29.

[2] - "Analyzing impact factors on composite services," in IEEE International Conference on Services Computing (SCC '09), sept. 2009, pp. $218-226$.

[3] B. Wetzstein, P. Leitner, F. Rosenberg, I. Brandic, S. Dustdar, and F. Leymann, "Monitoring and analyzing influential factors of business process performance," in 13th International Enterprise Distributed $\mathrm{Ob}$ ject Computing Conference (EDOC '09), 2009, pp. 141-150.

[4] P. Leitner, B. Wetzstein, F. Rosenberg, A. Michlmayr, S. Dustdar, and F. Leymann, "Runtime prediction of service level agreement violations for composite services," in International Conference on Service-Oriented Computing (ICSOC/ServiceWave'09), 2009, pp. 176-186.

[5] P. Leitner, A. Michlmayr, F. Rosenberg, and S. Dustdar, "Monitoring, prediction and prevention of sla violations in composite services," in IEEE International Conference on Web Services (ICWS'10), july 2010.

[6] E. Schmieders and A. Metzger, "Preventing performance violations of service compositions using assumption-based run-time verification," in 4th European Conference ServiceWave, 2011, pp. 194-205.

[7] L. Zeng, B. Benatallah, A. H.H. Ngu, M. Dumas, J. Kalagnanam, and H. Chang, "Qos-aware middleware for web services composition," IEEE Trans. Softw. Eng., vol. 30, pp. 311-327, May 2004.

[8] J. Kephart and D. Chess, "The vision of autonomic computing," Computer, vol. 36, no. 1, pp. $41-50$, jan 2003.

[9] J. Hielscher, R. Kazhamiakin, A. Metzger, and M. Pistore, "A framework for proactive self-adaptation of service-based applications based on online testing," in 1st European Conference ServiceWave, 2008.

[10] D. Dranidis, A. Metzger, and D. Kourtesis, "Enabling proactive adaptation through just-in-time testing of conversational services," in 3rd European Conference ServiceWave, 2010, pp. 63-75.

[11] V. Hodge and J. Austin, "A survey of outlier detection methodologies," Artif. Intell. Rev., vol. 22, no. 2, pp. 85-126, Oct. 2004.

[12] M. Jaeger, G. Rojec-Goldmann, and G. Muhl, "Qos aggregation for web service composition using workflow patterns," in 8th International Enterprise Distributed Object Computing Conference (EDOC), 2004.

[13] D. L. Cook, Program Evaluation and Review Technique-Applications in Education, 1966.

[14] M. Bramer, Principles of Data Mining. Springer, 2007.

[15] M. Hall, E. Frank, G. Holmes, B. Pfahringer, P. Reutemann, and I. H. Witten, "The weka data mining software: an update," SIGKDD Explor. Newsl., 2009.

[16] Z. Zheng, Y. Zhang, and M. Lyu, "Distributed qos evaluation for realworld web services," in 8th International Conference on Web Services (ICWS'10), july 2010, pp. $83-90$.

[17] F. Salfner, M. Lenk, and M. Malek, "A survey of online failure prediction methods," ACM Comput. Surv., vol. 42, pp. 10:1-10:42, March 2010. 